This is a postprint version of an article published as Order 23 (2006) 129-142.

DOI: $10.1007 /$ s11083-006-9036-y.

The final publication is available at link.springer.com.

\title{
DESCENDING CHAINS AND ANTICHAINS OF THE UNARY, LINEAR, AND MONOTONE SUBFUNCTION RELATIONS
}

\author{
ERKKO LEHTONEN
}

\begin{abstract}
The $\mathcal{C}$-subfunction relations on the set of operations on a finite base set $A$ defined by function classes $\mathcal{C}$ are examined. For certain clones $\mathcal{C}$ on $A$, it is determined whether the partial orders induced by the respective $\mathcal{C}$-subfunction relations have infinite descending chains or infinite antichains. More specifically, we investigate the subfunction relations defined by the clone of all functions on $A$, the clones of essentially at most unary operations, the clones of linear functions on a finite field, and the clones of monotone functions with respect to the various partial orders on $A$.
\end{abstract}

\section{INTRODUCTION}

We introduced in [10] the notion of $\mathcal{C}$-subfunction: for an arbitrary class $\mathcal{C}$ of functions on a fixed nonempty base set $A$, a function $f$ is called a $\mathcal{C}$-subfunction of a function $g$, if $f=g\left(h_{1}, \ldots, h_{n}\right)$ for some $h_{1}, \ldots, h_{n} \in \mathcal{C}$. This generalizes in a natural way the various notions of minors and subfunctions presented by Pippenger [12], Wang [20], Zverovich [21], and others, which actually correspond to the $\mathcal{C}$ subfunctions defined by some of the smallest clones on $A$, containing only projection maps, constant functions, or other essentially unary functions. These first examples of $\mathcal{C}$-subfunction relations satisfy the descending chain condition, as can easily be seen by a simple argument on essential arity. Such an argument is not applicable when the defining class $\mathcal{C}$ contains functions of higher essential arity, but we would like to know whether the descending chain condition is still satisfied.

We investigated the $\mathcal{C}$-subfunction relations between Boolean functions in [10]. In particular, we determined for every clone $\mathcal{C}$ of Boolean functions, whether the induced partial order has infinite descending chains or infinite antichains.

We now develop the concept in the more general setting of functions on an arbitrary nonempty finite base set $A$. In lack of a handy tool such as the Post lattice [13], there is little hope of obtaining such a complete and definitive answer in the general case as we have in the Boolean case. We must focus our analysis on some clones of interest.

This paper is organized as follows. We first introduce our basic definitions and notation in Section 2 . In subsequent sections, we then analyze certain $\mathcal{C}$-subfunction relations: the relations defined by the clone of all functions on $A$ in Section 3, clones of essentially at most unary functions in Section 4 , clones of linear functions on a

Key words and phrases. clones, subfunctions, composition of operations, partial orders, linear functions, monotone functions, Menger systems, Green's relations. 
finite field in Section 5, and clones of monotone functions with respect to a partial order in Section 6. Concluding remarks are made in Section 7.

\section{Definitions AND NOTATION}

2.1. Partially ordered sets. We recall standard terminology and notation that may be found in any textbook on orders and lattices (see, e.g., [1, 3]). A partially ordered set, or a poset, is a pair $(P, \leq)$, where $\leq$ is a partial order relation (i.e., a reflexive, transitive, and antisymmetric relation) on a nonempty set $P$. When $Q$ is a nonempty subset of $P$, the restriction of $\leq$ to $Q$ is a partial order relation on $Q$, and we call $\left(Q, \leq\left.\right|_{Q}\right)$ a subposet of $(P, \leq)$. We try to avoid the cumbersome notation for restrictions of relations and will denote $\leq\left.\right|_{Q}$ simply by $\leq$. We also refer to a poset $(P, \leq)$ simply as $P$.

A chain, or a totally ordered set, is a poset where all elements are pairwise comparable. An antichain is a poset where all elements are pairwise incomparable. We denote a chain of 2 elements by 2 .

The direct product of posets $P$ and $Q$, denoted $P \times Q$, is the poset $(P \times Q, \leq)$, where $(p, q) \leq\left(p^{\prime}, q^{\prime}\right)$ in $P \times Q$ if and only if both $p \leq p^{\prime}$ in $P$ and $q \leq q^{\prime}$ in $Q$. We also denote

$$
P^{n}=\underbrace{P \times \cdots \times P}_{n} .
$$

A poset is bounded if it has both a least element and a greatest element. A lattice is a poset $L$ in which any two elements $a$ and $b$ have a least upper bound $a \vee b$ and a greatest lower bound $a \wedge b$. A complete lattice is a poset in which every nonempty subset has a supremum and an infimum. A complete lattice is a lattice.

2.2. Functions and clones. Let $A$ be a nonempty set. A function (or an operation) on $A$ is a mapping $f: A^{n} \rightarrow A$ for some positive integer $n$, called the arity of $f$. We denote by $\mathcal{O}_{A}$ the set of all functions on $A$. The range, or image, of $f$ is the set $\operatorname{Im} f=\left\{f(\mathbf{a}): \mathbf{a} \in A^{n}\right\}$. The kernel of $f$ is the equivalence relation $\operatorname{ker} f=\left\{(\mathbf{a}, \mathbf{b}) \in A^{n} \times A^{n}: f(\mathbf{a})=f(\mathbf{b})\right\}$ on the domain $A^{n}$ of $f$.

For a fixed arity $n$, and for $1 \leq i \leq n$, the $i$ th projection, denoted $x_{i}^{n}$, is the function $\left(a_{1}, \ldots, a_{n}\right) \mapsto a_{i}$. The $n$-ary constant function having value $a \in A$ everywhere is denoted by $\widehat{a}^{n}$. Whenever the arity is clear from the context, we omit the superscripts indicating arity.

For $1 \leq i \leq n$, we say that the $i$ th variable is essential in $f$, if there are points $\mathbf{a}=\left(a_{1}, \ldots, a_{n}\right), \mathbf{a}^{\prime}=\left(a_{1}^{\prime}, \ldots, a_{n}^{\prime}\right)$ such that $a_{i} \neq a_{i}^{\prime}$ and $a_{j}=a_{j}^{\prime}$ for all $j \neq i$ and $f(\mathbf{a}) \neq f\left(\mathbf{a}^{\prime}\right)$. If a variable is not essential in $f$, then it is inessential in $f$. The essential arity of $f$, denoted Ess $f$, is the number of essential variables in $f$.

If $f$ is an $n$-ary function (the outer function) and $g_{1}, \ldots, g_{n}$ are all $m$-ary functions (the inner functions), then the composition (or superposition) of $f$ with $g_{1}, \ldots, g_{n}$, denoted $f\left(g_{1}, \ldots, g_{n}\right)$, is an $m$-ary function, and its value on $\mathbf{a} \in A^{m}$ is $f\left(g_{1}(\mathbf{a}), \ldots, g_{n}(\mathbf{a})\right)$. This is equivalent to the composition $f \circ g$, where the mapping $g: A^{m} \rightarrow A^{n}$ is defined as $g(\mathbf{a})=\left(g_{1}(\mathbf{a}), \ldots, g_{n}(\mathbf{a})\right)$, which we simply denote by $g=\left(g_{1}, \ldots, g_{n}\right)$.

A class is a subset $\mathcal{C} \subseteq \mathcal{O}_{A}$. The $n$-ary part of a class $\mathcal{C}$, denoted by $\mathcal{C}^{(n)}$, is the set of $n$-ary functions in $\mathcal{C}$. A clone on $A$ is a class $\mathcal{C} \subseteq \mathcal{O}_{A}$ that contains all projections and is closed under functional composition (i.e., if $f, g_{1}, \ldots, g_{n} \in \mathcal{C}$, then $f\left(g_{1}, \ldots, g_{n}\right) \in \mathcal{C}$ whenever the composition is defined). We denote by $\langle\mathcal{C}\rangle$ 
the clone generated by $\mathcal{C}$. The clones on $A$ constitute an inclusion-ordered lattice, denoted $\mathcal{L}_{A}$, where the lattice operations are the following: meet is the intersection, join is the smallest clone containing the union. See [19] for discussion on clones.

2.3. $\mathcal{C}$-subfunctions. We consider functions on a fixed nonempty set $A$. Let $\mathcal{C}$ be a class of functions. We say that a function $f$ is a $\mathcal{C}$-subfunction of a function $g$, denoted $f \preceq_{\mathcal{C}} g$, if $f=g\left(h_{1}, \ldots, h_{m}\right)$ for some $h_{1}, \ldots, h_{m} \in \mathcal{C}$. If $f$ and $g$ are $\mathcal{C}$-subfunctions of each other, we say they are $\mathcal{C}$-equivalent and denote $f \equiv_{\mathcal{C}} g$. If $f \preceq_{\mathcal{C}} g$ but $g \swarrow_{\mathcal{C}} f$, we say that $f$ is a proper $\mathcal{C}$-subfunction of $g$ and denote $f \prec_{\mathcal{C}} g$. If both $f \swarrow_{\mathcal{C}} g$ and $g \swarrow_{\mathcal{C}} f$, we say that $f$ and $g$ are $\mathcal{C}$-incomparable and denote $f \|_{\mathcal{C}} g$. If the class $\mathcal{C}$ is clear from the context, we may omit the subscripts indicating the class. We have now defined families of binary relations $\preceq_{\mathcal{C}}$ and $\equiv_{\mathcal{C}}$ on $\mathcal{O}_{A}$, indexed by the class $\mathcal{C}$. Most of the basic results that were proved in [10] for a two-element base set are straightforwardly generalized for arbitrary base sets; we just state these facts and do not repeat the detailed proofs here.

For any class $\mathcal{C}$, the set of all $\mathcal{C}$-subfunctions of $x_{1}$ equals $\mathcal{C}$, and therefore the relations $\preceq_{\mathcal{C}}$ and $\preceq_{\mathcal{K}}$ are always distinct for $\mathcal{C} \neq \mathcal{K}$. Also, for any classes $\mathcal{C}$ and $\mathcal{K}$, $\preceq_{\mathcal{C}}$ is a subrelation of $\preceq_{\mathcal{K}}$ if and only if $\mathcal{C} \subseteq \mathcal{K}$.

The $\mathcal{C}$-subfunction relation $\preceq_{\mathcal{C}}$ is reflexive if and only if the class $\mathcal{C}$ contains all projections; and $\preceq_{\mathcal{C}}$ is transitive if and only if $\mathcal{C}$ is closed under functional composition. Hence, $\preceq_{\mathcal{C}}$ is a preorder on $\mathcal{O}_{A}$ if and only if $\mathcal{C}$ is a clone. If $\mathcal{C}$ is a clone, then $\equiv_{\mathcal{C}}$ is an equivalence relation, and the $\mathcal{C}$-equivalence class of $f$ is denoted by $[f]_{\mathcal{C}}$. As for preorders, $\preceq_{\mathcal{C}}$ induces a partial order $\leq_{\mathcal{C}}$ on $\mathcal{O}_{A} / \equiv_{\mathcal{C}}$.

It is clear that $\operatorname{Im} f \subseteq \operatorname{Im} g$ for any $f \preceq c g$ and any $\mathcal{C}$. Therefore, any $\mathcal{C}$ equivalent functions have the same range. This implies in particular that for any element $a \in A$, the constant functions $\widehat{a}$ of all arities form a $\mathcal{C}$-equivalence class for any clone $\mathcal{C}$, and these classes are minimal elements in the partial order $\leq_{\mathcal{C}}$ on $\mathcal{O}_{A} / \equiv_{\mathcal{C}}$. For any clones $\mathcal{C}$ and $\mathcal{K}, \equiv_{\mathcal{C}}$ is a subrelation of $\equiv_{\mathcal{K}}$ whenever $\mathcal{C} \subseteq \mathcal{K}$.

We still point out that whenever there is an infinite antichain of $\mathcal{C}$-incomparable functions, we also have an infinite antichain of $\mathcal{K}$-incomparable functions for every subclone $\mathcal{K}$ of $\mathcal{C}$.

2.4. The current problem. Given a clone $\mathcal{C}$ on $A$, we are interested in the ordertheoretical properties of the preorder $\preceq_{\mathcal{C}}$ on $\mathcal{O}_{A}$ and the induced partial order $\leq_{\mathcal{C}}$ on $\mathcal{O}_{A} / \equiv_{\mathcal{C}}$. Two questions in particular arise immediately: Are there infinite descending chains of $\mathcal{C}$-subfunctions? How large antichains of $\mathcal{C}$-incomparable functions are there?

The case of a singleton base set $A$ is trivial, because we only have one clone, namely the clone $\mathcal{O}_{A}$ of all functions, and all functions are $\mathcal{O}_{A}$-equivalent. These questions were resolved for all clones on the two-element base set $A=\{0,1\}$ in [10]. There is little hope of giving such a complete and definite answer in the more general case, because there are uncountably many clones on $A$ to begin with, and the structure of the lattice $\mathcal{L}_{A}$ of clones on $A$ is only partially known when $|A| \geq 3$. In the more general case, there is no handy tool such as the Post lattice [13] at one's disposal, and one must confine the analysis to certain interesting clones.

In what follows, we assume that the base set $A$ is finite and $|A|=k \geq 3$. Because it is unimportant what the elements of the base set are, we assume, without loss of generality, that $A=\{0,1, \ldots, k-1\}=\underline{k}$. 
In the following sections, we will analyze the $\mathcal{C}$-subfunction relations in the cases when the defining class $\mathcal{C}$ is the clone of all functions on $A$, a clone containing only essentially at most unary functions, a clone of linear functions on a finite field, or a clone of monotone functions with respect to a partial order on $A$.

\section{The ClONE OF ALL FUnCtions}

3.1. Green's relations on Menger systems. Suppose that $I$ is a nonempty set of positive integers, $\left(M_{n}\right)_{n \in I}$ is a family of nonempty pairwise disjoint sets, and $\left(o_{n}\right)_{n \in I}$ is a family of partial $(n+1)$-ary operations on $M=\bigcup_{n \in I} M_{n}$ which satisfy the following conditions

(1) if $x \in M_{n}, y_{1}, \ldots, y_{n} \in M_{m}$, then $o_{n}\left(x, y_{1}, \ldots, y_{n}\right)$ is defined and $o_{n}\left(x, y_{1}, \ldots, y_{n}\right) \in$ $M_{m}$

(2) for all $l, m, n \in I, x \in M_{l}, y_{1}, \ldots, y_{l} \in M_{n}, z_{1}, \ldots, z_{n} \in M_{m}$, the following superassociativity property holds:

$$
\begin{aligned}
o_{n}\left(o_{l}\left(x, y_{1}, \ldots, y_{l}\right), z_{1}, \ldots, z_{n}\right) & \\
& =o_{l}\left(x, o_{n}\left(y_{1}, z_{1}, \ldots, z_{n}\right), \ldots, o_{n}\left(y_{l}, z_{1}, \ldots, z_{n}\right)\right) .
\end{aligned}
$$

Then the system $\mathbf{M}=\left(\left(M_{n}\right)_{n \in I} ;\left(o_{n}\right)_{n \in I}\right)$ is called a Menger system.

A Menger system is called unitary, if for every $n \in I$ there exist elements $e_{1}^{n}, \ldots, e_{n}^{n} \in M_{n}$ (called selectors) which satisfy the identities

$$
\begin{gathered}
o_{n}\left(x, e_{1}^{n}, \ldots, e_{n}^{n}\right)=x, \\
o_{n}\left(e_{i}^{n}, y_{1}, \ldots, y_{n}\right)=y_{i},
\end{gathered}
$$

for all $x \in M_{n}, y_{1}, \ldots, y_{n} \in M_{m}$.

For a family $\left(\mathcal{O}_{A}^{(n)}\right)_{n \in I}$ of sets of all $n$-ary functions on $A$, superposition can be expressed by a family $\left(o_{n}\right)_{n \in I}$ of partial $(n+1)$-ary operations, and it is easy to see that the system $\left(\left(\mathcal{O}_{A}^{(n)}\right)_{n \in I} ;\left(o_{n}\right)_{n \in I}\right)$ is a unitary Menger system; its selectors are the various projection maps $x_{i}^{n}$ for $1 \leq i \leq n$. Such a system is called a full function system.

Menger systems are a generalization of Menger algebras [11]. For further information on Menger systems and other algebras of multiplace functions, we refer the reader to the survey article by Schein and Trohimenko [16].

Green [4] introduced certain equivalence relations on a semigroup that have played fundamental role in semigroup theory; see, e.g., [7]. Analogs of Green's equivalences $\mathcal{L}, \mathcal{R}, \mathcal{D}$, and $\mathcal{H}$ for Menger systems were defined by J. Henno [5] (see also [6]) in the following way:

- $a \mathcal{L} b\left(a \in M_{n}, b \in M_{m}, n, m \in I\right)$ if and only if $a=b$ or there exist $s_{1}, \ldots, s_{n} \in$ $M_{m}, t_{1}, \ldots, t_{m} \in M_{n}$ such that $a=o_{m}\left(b, t_{1}, \ldots, t_{m}\right), b=o_{n}\left(a, s_{1}, \ldots, s_{n}\right)$;

- $a \mathcal{R} b$ if and only if $a, b \in M_{n}$ for some $n \in I$ and $a=b$ or there exist $s \in M_{m}$, $t \in M_{k}, m, k \in I$ such that $a=o_{k}(t, b, \ldots, b), b=o_{m}(s, a, \ldots, a)$;

- $\mathcal{D}=\mathcal{L} \cup \mathcal{R} ; \mathcal{H}=\mathcal{L} \cap \mathcal{R}$.

Define for $a \in M_{n}, b \in M_{m}, n, m \in I$ the binary relations $\leq_{L}, \leq_{R}, \leq_{H}$ by

- $a \leq_{L} b$ if and only if $a=b$ or $a=o_{m}\left(b, t_{1}, \ldots, t_{m}\right)$ for some $t_{1}, \ldots, t_{m} \in M_{n}$;

- $a \leq_{R} b$ if and only if $a=b$ or $a=o_{k}(t, b, \ldots, b)$ for some $t \in M_{k}, k \in I$;

- $a \leq_{H} b$ if and only if $a \leq_{L} b$ and $a \leq_{R} b$.

All these relations are preorders, and by definition, $a \mathcal{L} b$ if and only if $a \leq_{L} b$ and $b \leq_{L} a$ (and similarly for $\mathcal{R}$ and $\mathcal{H}$ ).

Henno [5] proved the following for Green's relations on full function systems. 
Theorem 3.1. For arbitrary $f \in \mathcal{O}_{A}^{(n)}, g \in \mathcal{O}_{A}^{(m)}$ :

(i) $f \mathcal{L} g$ if and only if $\operatorname{Im} f=\operatorname{Im} g$;

(ii) $f \mathcal{R} g$ if and only if $n=m$ and $\operatorname{ker} f=\operatorname{ker} g$;

(iii) $f \mathcal{D} g$ if and only if $|\operatorname{Im} f|=|\operatorname{Im} g|$;

(iv) $f \mathcal{H} g$ if and only if $n=m$, $\operatorname{ker} f=\operatorname{ker} g$, and $\operatorname{Im} f=\operatorname{Im} g$.

3.2. $\mathcal{O}_{A}$-subfunctions. The $\mathcal{O}_{A}$-subfunction relation $\preceq_{\mathcal{O}_{A}}$ on $\mathcal{O}_{A}$ is in fact the same as Green's preorder $\leq_{L}$ on the full function system $\left(\left(\mathcal{O}_{A}^{(n)}\right)_{n \in N} ;\left(o_{n}\right)_{n \in N}\right)$, where $N$ denotes the set of positive integers. Henno [5, Lemma 10] showed that $f \leq_{L} g$ if and only if $\operatorname{Im} f \subseteq \operatorname{Im} g$, and Theorem 3.1 characterizes the $\mathcal{O}_{A^{-}}$ equivalence classes completely: $f \equiv_{\mathcal{O}_{A}} g$ if and only if $\operatorname{Im} f=\operatorname{Im} g$.

Theorem 3.2. The poset $\left(\mathcal{O}_{A} / \equiv_{\mathcal{O}_{A}}, \leq_{\mathcal{O}_{A}}\right)$ is isomorphic to the poset $(\mathcal{P}(A) \backslash$ $\{\emptyset\}, \subseteq)$ of nonempty subsets of $A$, ordered by inclusion. The largest chain of this poset has $k$ elements, and the largest antichain has $\left(\begin{array}{c}k \\ \lfloor k / 2\rfloor\end{array}\right)$ elements.

Proof. The isomorphicity of the posets follows from the above observations, and the size of the largest chain is obvious. The largest antichain has $\left(\begin{array}{c}k \\ \lfloor k / 2\rfloor\end{array}\right)$ elements by Sperner's theorem [18].

\section{The ClONES OF ESSENTIALly AT MOST UNARY FUnCTIONS}

Is this section, we assume that $\mathcal{C}=\langle M\rangle$ for some transformation monoid $M$ on $A$, i.e., $\mathcal{C}$ contains only essentially at most unary functions.

We denote by $\mathcal{J}$ the clone of all projections. Following the guidelines of [10], we observe that the $\mathcal{J}$-subfuctions of $f$ are exactly those functions that can be obtained from $f$ by repeated permutation and identification of variables, cylindrification, and deletion of inessential variables; and any $\mathcal{J}$-equivalent functions can be obtained from each other by permutation of variables, cylindrification, and deletion of inessential variables. It is clear that every nonconstant function $f$ is $\mathcal{J}$-equivalent (and hence $\mathcal{C}$-equivalent for every clone $\mathcal{C}$ ) to the function $f^{\text {ess }}$ of arity Ess $f$, obtained by deleting all inessential variables from $f$. We also agree that for every $a \in A, n \geq 1,\left(\widehat{a}^{n}\right)^{\text {ess }}=\widehat{a}^{1}$.

Lemma 4.1. Let $M$ be a transformation monoid on $A$. If $f \preceq\langle M\rangle g$, then Ess $f \leq$ Ess $g$.

Proof. Let $f=g\left(h_{1}, \ldots, h_{m}\right)$ for some $h_{1}, \ldots, h_{m} \in\langle M\rangle$. Each essential variable of $f$ has to be essential in at least one of the inner functions $h_{i}$ substituted for an essential variable of $g$. Since the $h_{i}$ 's are essentially at most unary, it is clear that Ess $f \leq \operatorname{Ess} g$

Theorem 4.2. For any transformation monoid $M$, there is no infinite descending chain of $\langle M\rangle$-subfunctions.

Proof. Suppose, on the contrary, that there is an infinite descending chain

$$
f_{1} \succ_{\langle M\rangle} f_{2} \succ_{\langle M\rangle} f_{3} \succ_{\langle M\rangle} \cdots \text {. }
$$

Since each $f_{i}$ is $\mathcal{J}$-equivalent to $f_{i}^{\text {ess }}$, we can assume that all variables are essential in each $f_{i}$. Lemma 4.1 implies that there is a $j$ such that all functions $f_{i}$ with $i \geq j$ have the same arity. We have reached a contradiction, because there are only a finite number of functions of any fixed arity. 
Denote by $\mathcal{U}=\left\langle\mathcal{O}_{A}^{(1)}\right\rangle$ the clone of all essentially at most unary functions on $A$. In order to show that there is an infinite antichain of $\mathcal{U}$-incomparable functions, we generalize the construction presented by Pippenger [12, Proposition 3.4], and we rephrase his proof in the language of subfunctions and functional composition. For $n \geq 4$, define the $n$-ary function $\alpha_{n}$ as

$$
\alpha_{n}(\mathbf{a})= \begin{cases}1, & \text { if }\left|\left\{i: a_{i}=1\right\}\right| \in\{1, n-1\} \\ 0, & \text { otherwise }\end{cases}
$$

Proposition 4.3. $\alpha_{m} \|_{\mathcal{U}} \alpha_{n}$ for $n \neq m$.

Proof. We call a function $f$ poor, if $f=\widehat{1}$ or $f$ does not take on value 1 . A function is rich, if it is not poor. We make a few observations on the composition $\alpha_{n}\left(\gamma_{1}, \ldots, \gamma_{n}\right)$, where $\gamma_{i} \in \mathcal{U}$. First, if at least two of the inner functions are $\widehat{1}$ and at least two of them do not take on value 1 , then $\alpha_{n}\left(\gamma_{1}, \ldots, \gamma_{n}\right)=\widehat{0}$. Second, if at most three of the inner functions are poor, then $\alpha_{n}\left(\gamma_{1}, \ldots, \gamma_{n}\right)$ is not a constant function, taking on both values 0 and 1 . Third, if a variable is essential only in poor inner functions, then it is inessential in the composition $\alpha_{n}\left(\gamma_{1}, \ldots, \gamma_{n}\right)$.

Assume that $n<m$. Since for any $i$, all $i$ variables are essential in $\alpha_{i}$, we clearly have that $\alpha_{m} \npreceq \mathcal{U} \alpha_{n}$ by Lemma 4.1.

Suppose, on the contrary, that $\alpha_{n} \preceq_{\mathcal{U}} \alpha_{m}$. Then $\alpha_{n}=\alpha_{m}\left(\gamma_{1}, \ldots, \gamma_{m}\right)$ for some $\gamma_{i} \in \mathcal{U}$. Since all $n$ variables are essential in $\alpha_{n}$, we must have that for $i=1, \ldots, n$, there is a rich inner function with the $i$ th variable essential.

Suppose first that for each $i$, the $i$ th variable is essential in exactly one of the inner functions. Since $n<m$, at least one of the inner functions is poor, say $\gamma_{p}$. Let $\gamma_{q}, \gamma_{r}, \gamma_{s}$ be distinct rich inner functions with their $q^{\prime}$-th, $r^{\prime}$-th, $s^{\prime}$-th variables essential, respectively. For $1 \leq i \leq n, i \notin\left\{q^{\prime}, r^{\prime}, s^{\prime}\right\}$, let $h_{i}=x_{i}$ and let $h_{q^{\prime}}=\widehat{c_{1}}, h_{r^{\prime}}=\widehat{c_{2}}, h_{s^{\prime}}=\widehat{c_{3}}$ for some appropriately chosen constant functions such that, denoting $\gamma_{i}^{\prime}=\gamma_{i}\left(h_{1}, \ldots, h_{n}\right)$, exactly two of $\gamma_{p}^{\prime}, \gamma_{q}^{\prime}, \gamma_{r}^{\prime}, \gamma_{s}^{\prime}$ equal the constant function $\widehat{1}$ and the other two do not take on value 1 . By our initial observations, we have that $\alpha_{m}\left(\gamma_{1}^{\prime}, \ldots, \gamma_{m}^{\prime}\right)=\widehat{0}$ and, on the other hand,

$$
\begin{aligned}
\alpha_{m}\left(\gamma_{1}^{\prime}, \ldots, \gamma_{m}^{\prime}\right) & =\alpha_{m}\left(\gamma_{1}\left(h_{1}, \ldots, h_{m}\right), \ldots, \gamma_{m}\left(h_{1}, \ldots, h_{m}\right)\right) \\
& =\left(\alpha_{m}\left(\gamma_{1}, \ldots, \gamma_{m}\right)\right)\left(h_{1}, \ldots, h_{n}\right)=\alpha_{n}\left(h_{1}, \ldots, h_{n}\right),
\end{aligned}
$$

which is not a constant function, a contradiction.

Suppose then that for some $j$, the $j$ th variable is essential in at least two rich inner functions. Let $1 \leq j_{1}<j_{2} \leq n$ such that $j \notin\left\{j_{1}, j_{2}\right\}$. For $i \notin\left\{j, j_{1}, j_{2}\right\}$, let $h_{i}=x_{i}$ and let $h_{j}, h_{j_{1}}, h_{j_{2}}$ be suitably chosen constant functions such that, denoting $\gamma_{i}^{\prime}=\gamma_{i}\left(h_{1}, \ldots, h_{n}\right)$, among $\gamma_{1}^{\prime}, \ldots, \gamma_{m}^{\prime}$, there are at least two $\widehat{0}$ 's and at least two functions not taking on value 1 . By our observations, we have again that $\alpha_{m}\left(\gamma_{1}^{\prime}, \ldots, \gamma_{m}^{\prime}\right)=\widehat{0}$ and, on the other hand, $\alpha_{m}\left(\gamma_{1}^{\prime}, \ldots, \gamma_{m}^{\prime}\right)=\alpha_{n}\left(h_{1}, \ldots, h_{n}\right)$, which is not a constant function, a contradiction.

Of course, $\mathcal{U}$-incomparable functions are $\mathcal{C}$-incomparable for every subclone $\mathcal{C}$ of $\mathcal{U}$. We have established the following.

Theorem 4.4. For every transformation monoid $M$ on $A$, there is an infinite antichain of $\langle M\rangle$-incomparable functions. 


\section{ThE ClONES OF LINEAR FUNCTIONS ON FINITE FIELDS}

5.1. Functional independence. For a class $\mathcal{C}$ of functions, we say that $m$-ary functions $g_{1}, \ldots, g_{n}$ are $\mathcal{C}$-dependent, if there is an $i$ and an $(n-1)$-ary function $h \in \mathcal{C}$ such that $g_{i}=h\left(g_{1}, \ldots, g_{i-1}, g_{i+1}, \ldots, g_{n}\right)$. If the functions $g_{1}, \ldots, g_{n}$ are not $\mathcal{C}$-dependent, they are $\mathcal{C}$-independent. We say that functions are functionally dependent, if they are $\mathcal{O}_{A}$-dependent; and we say that functions are functionally independent, if they are $\mathcal{O}_{A}$-independent. It is clear that $\mathcal{C}$-independence implies $\mathcal{K}$-independence for every $\mathcal{K} \subseteq \mathcal{C}$.

Let $\mathcal{C}$ be a clone and let $f$ be any function. Consider representations of $f$ as a composition $f=\phi\left(g_{1}, \ldots, g_{l}\right)$, where $g_{1}, \ldots, g_{l} \in \mathcal{C}$ and $\phi \in \mathcal{O}_{A}$. Such a representation is called a $\mathcal{C}$-decomposition of $f$. There always exists $\mathcal{C}$-decompositions for any function $f$, because $f=f\left(x_{1}, \ldots, x_{n}\right)$ and projections are members of all clones. For a nonconstant function $f$, a $\mathcal{C}$-decomposition of $f$ with the smallest possible number $l$ of inner functions (or the smallest possible arity of the outer function) is called minimal, and this smallest number is called the $\mathcal{C}$-degree of $f$, $\operatorname{denoted} \operatorname{deg}_{\mathcal{C}} f$. We clearly have that $\operatorname{deg}_{\mathcal{C}} f \leq \operatorname{Ess} f$. For constant functions $f$, we agree that $\operatorname{deg}_{\mathcal{C}} f=0$.

Lemma 5.1. If $\mathcal{C} \subseteq \mathcal{K}$, then $\operatorname{deg}_{\mathcal{K}} f \leq \operatorname{deg}_{\mathcal{C}} f$.

Proof. Every $\mathcal{C}$-decomposition of $f$ is also a $\mathcal{K}$-decomposition, not necessarily minimal.

Lemma 5.2. If $f \preceq_{\mathcal{C}} g$, then $\operatorname{deg}_{\mathcal{C}} f \leq \operatorname{deg}_{\mathcal{C}} g$.

Proof. Let $f=g\left(h_{1}, \ldots, h_{m}\right)$ for some $h_{1}, \ldots, h_{m} \in \mathcal{C}$, and let $g=\gamma\left(k_{1}, \ldots, k_{l}\right)$ be a minimal $\mathcal{C}$-decomposition. Then $f=\gamma\left(k_{1}, \ldots, k_{l}\right)\left(h_{1}, \ldots, h_{n}\right)=\gamma\left(k_{1}^{\prime}, \ldots, k_{l}^{\prime}\right)$, where $k_{i}^{\prime}=k_{i}\left(h_{1}, \ldots, h_{n}\right)$. This is a $\mathcal{C}$-decomposition of $f$, but not necessarily a minimal one, so $\operatorname{deg}_{\mathcal{C}} f \leq l=\operatorname{deg}_{\mathcal{C}} g$.

Corollary 5.3. If $f \equiv_{\mathcal{C}} g$, then $\operatorname{deg}_{\mathcal{C}} f=\operatorname{deg}_{\mathcal{C}} g$.

Lemma 5.4. In a minimal $\mathcal{C}$-decomposition, the inner functions are functionally independent.

Proof. Let $f=\phi\left(g_{1}, \ldots, g_{l}\right)$ be a minimal $\mathcal{C}$-decomposition. Suppose, on the contrary, that the inner functions are functionally dependent, i.e., there is an $i$ such that $g_{i}=\gamma\left(g_{1}, \ldots, g_{i-1}, g_{i+1}, \ldots, g_{l}\right)$. But then

$$
\begin{aligned}
f & =\phi\left(g_{1}, \ldots, g_{i-1}, \gamma\left(g_{1}, \ldots, g_{i-1}, g_{i+1}, \ldots, g_{l}\right), g_{i+1}, \ldots, g_{l}\right) \\
& =\phi\left(x_{1}, \ldots, x_{i-1}, \gamma, x_{i}, \ldots, x_{l-1}\right)\left(g_{1}, \ldots, g_{i-1}, g_{i+1}, \ldots, g_{l}\right),
\end{aligned}
$$

contradicting the minimality of the given $\mathcal{C}$-decomposition.

5.2. Descending chains. Assume that the base set $A$ has a field structure $(A,+, \cdot)$, and assume, without loss of generality, that 0 and 1 are the respective zero and identity elements. We recall that both $A^{n}$ and $A^{A^{n}}$ are vector spaces over the field $(A,+, \cdot)$. A function $f \in A^{A^{n}}$ is linear (or affine, if one prefers), if it is of the form $f=a_{1} x_{1}+\cdots+a_{n} x_{n}+\widehat{c}$ for some $a_{1}, \ldots, a_{n}, c \in A$. In such a representation, we call $\sum_{i=1}^{n} a_{i} x_{i}$ the linear part and $\widehat{c}$ the constant part of $f$. We denote by $L$ the class of all linear functions, which is a maximal clone according to Rosenberg's renowned classification [15]. 
Proposition 5.5. Assume that $f=g\left(\phi_{1}, \ldots, \phi_{d}\right)$ is a minimal L-decomposition. Then $f \equiv_{L} g$.

Proof. Assume that $f$ is $n$-ary. It is clear that $f \preceq_{L} g$. We show that $g \preceq_{L} f$. By Lemma 5.4, the inner functions $\phi_{1}, \ldots \phi_{d}$ are functionally independent, and they have the form $\phi_{i}=\sum_{j=1}^{n} b_{i j} x_{j}+\widehat{c_{i}}=\lambda_{i}+\widehat{c_{i}}$. The linear parts $\lambda_{1}, \ldots, \lambda_{d}$ are linearly independent (with the common meaning of the term). For, suppose, on the contrary, that the linear parts $\lambda_{1}, \ldots, \lambda_{d}$ are linearly dependent. Then there exist coefficients $a_{1}, \ldots, a_{d}$, not all zero, such that $\sum_{i=1}^{d} a_{i} \lambda_{i}=\widehat{0}$. If $a_{j} \neq 0$, then $a_{j} \lambda_{j}=-\sum_{i \neq j} a_{i} \lambda_{i}$, and so $\phi_{j}=\lambda_{j}+\widehat{c_{j}}=\widehat{c_{j}}-a_{j}^{-1} \sum_{i \neq j} a_{i}\left(\phi_{i}-\widehat{c_{i}}\right)$, a contradiction to the functional independence of $\phi_{1}, \ldots, \phi_{d}$.

Define the $d \times n$ matrix $B=\left(b_{i j}\right)$ and the $d$-vector $\mathbf{c}=\left(c_{1}, \ldots, c_{d}\right)^{\mathrm{T}}$. From elementary linear algebra we know that $B$ has a right inverse $E$ and the matrix equation $B \mathbf{y}=-\mathbf{c}$ has a solution $\mathbf{y}=\left(y_{1}, \ldots, y_{n}\right)^{\mathrm{T}}$ if and only if $B$ has full rank $d$. (The right inverse of $B$ is an $n \times d$ matrix $E$ such that $B E=I$, where $I$ is the $d \times d$ identity matrix.) Since the linear parts are linearly independent, this condition is satisfied. We regard the elements of $E$ and $\mathbf{y}$ as the coefficients of the linear functions $\psi_{1}, \ldots, \psi_{n}$, defined as

$$
\psi_{i}=e_{i 1} x_{1}+\cdots+e_{i d} x_{d}+\widehat{y_{i}} .
$$

We have that for $j=1, \ldots, d$,

$$
\begin{aligned}
\phi_{j}\left(\psi_{1}, \ldots, \psi_{n}\right) & =\widehat{c_{j}}+\sum_{i=1}^{n} b_{j i}\left(e_{i 1} x_{1}+\cdots+e_{i n} x_{d}+\widehat{y_{i}}\right) \\
& =\widehat{c_{j}}+\sum_{i=1}^{d} \sum_{k=1}^{n} b_{j k} e_{k i} x_{i}+\sum_{k=1}^{n} \widehat{b_{j k} y_{k}} \\
& =x_{j}+\widehat{c_{j}-c_{j}}=x_{j} .
\end{aligned}
$$

Thus,

$$
\begin{aligned}
f\left(\psi_{1}, \ldots, \psi_{n}\right) & =g\left(\phi_{1}, \ldots, \phi_{d}\right)\left(\psi_{1}, \ldots, \psi_{n}\right) \\
& =g\left(\phi_{1}\left(\psi_{1}, \ldots, \psi_{n}\right), \ldots, \phi_{d}\left(\psi_{1}, \ldots, \psi_{n}\right)\right) \\
& =g\left(x_{1}, \ldots, x_{d}\right)=g
\end{aligned}
$$

i.e., $g \preceq_{L} f$.

Proposition 5.6. Assume that $f \preceq_{L} g$. Then $f \equiv_{L} g$ if and only if $\operatorname{deg}_{L} f=$ $\operatorname{deg}_{L} g$.

Proof. By Corollary 5.3, $L$-equivalence implies equality of $L$-degrees.

Let $\operatorname{deg}_{L} g=d$, and let $g=\phi\left(\gamma_{1}, \ldots, \gamma_{d}\right)$ be a minimal $L$-decomposition. We have that $f=g\left(h_{1}, \ldots, h_{n}\right)$ for some $h_{1}, \ldots, h_{n} \in L$ and so $f=\phi\left(\gamma_{1}^{\prime}, \ldots, \gamma_{d}^{\prime}\right)$, where $\gamma_{i}^{\prime}=\gamma_{i}\left(h_{1}, \ldots, h_{n}\right) \in L$. Assuming that $\operatorname{deg}_{L} f=\operatorname{deg}_{L} g=d$, this is a minimal $L$-decomposition, and therefore $\gamma_{1}^{\prime}, \ldots, \gamma_{d}^{\prime}$ are functionally independent. By Proposition 5.5, both $f$ and $g$ are $L$-equivalent to $\phi$ and hence $L$-equivalent to each other.

We have now established the following.

Theorem 5.7. There is no infinite descending chain of L-subfunctions. 
Denote by $L_{0}$ the clone of linear functions with constant part $\widehat{0}$. We note that the proof of Proposition 5.5 is easily modified for the $L_{0}$-subfunctions: $\mathbf{0}$ is a solution of the equation $B \mathbf{y}=\mathbf{0}$, so the functions $\phi_{i}$ will be members of $L_{0}$.

Theorem 5.8. There is no infinite descending chain of $L_{0}$-subfunctions.

5.3. Antichains. In order to construct an infinite antichain of $L$-incomparable functions, we impose the total order $0 \leq 1 \leq \cdots \leq k-1$ on $A$. For $n \geq 4$, define the $n$-ary function $\alpha_{n}$ as

$$
\alpha_{n}(\mathbf{a})= \begin{cases}1, & \text { if } \mathbf{a} \in\{0,1\}^{n} \text { and } \\ & \left|\left\{i: a_{i}=1\right\}\right| \in\{1, n-1\}, \\ 0, & \text { if } \mathbf{a} \in\{0,1\}^{n} \text { and } \\ & \left|\left\{i: a_{i}=1\right\}\right| \notin\{1, n-1\}, \\ \max \left\{a_{1}, \ldots, a_{n}\right\}, & \text { if } \mathbf{a} \notin\{0,1\}^{n} .\end{cases}
$$

A unit vector is a vector in which exactly one component has value 1 and all other components have value 0 . Denote by $\mathbf{e}_{i}$ the unit vector whose $i$ th component has value 1 . For a vector $\mathbf{v} \in\{0,1\}^{n}$, denote by $\overline{\mathbf{v}}$ the complement of $\mathbf{v}$, defined as $\overline{\mathbf{v}}=\left(1-v_{1}, \ldots, 1-v_{n}\right)$. The number of nonzero elements in a vector $\mathbf{v}$ is called the Hamming weight of $\mathbf{v}$ and denoted by $w(\mathbf{v})$. We denote $\mathbf{0}=(0, \ldots, 0)$, $\mathbf{1}=(1, \ldots, 1)$.

Proposition 5.9. Let $(A,+, \cdot)$ be a field of characteristic $p$. The functions $\alpha_{i p+2}$ $(i \geq 2)$ are pairwise $L$-incomparable.

Proof. Suppose that $n \neq m$ and $\alpha_{n} \preceq_{L} \alpha_{m}$. There exist $n$-ary functions $h_{1}, \ldots, h_{n} \in$ $L$ such that $\alpha_{n}=\alpha_{m}\left(h_{1}, \ldots, h_{m}\right)$. Let $h_{i}=c_{1 i} x_{1}+\cdots+c_{n i} x_{n}+\widehat{d}_{i}$, and consider the $n \times m$ matrix $C=\left(c_{i j}\right)$ and the $m$-vector $\mathbf{d}=\left(d_{1}, \ldots, d_{m}\right)$. The mapping $h=\left(h_{1}, \ldots, h_{m}\right)$ can be described in terms of matrices over the field $A$ as $h(\mathbf{a})=\mathbf{a} C+\mathbf{d}$.

Since $\alpha_{n}(\mathbf{0})=0$, we have that $h(\mathbf{0})=\mathbf{d} \in\{0,1\}^{m}$ and $w(\mathbf{v}) \notin\{1, m-1\}$. We also observe that $h$ maps the elements of $\{0,1\}^{n}$ to elements of $\{0,1\}^{m}$ and, in particular, unit vectors and the complements of unit vectors to unit vectors and complements of unit vectors. Thus, there exists a mapping $\sigma:\{1, \ldots, n\} \rightarrow$ $\{1, \ldots, m\}$ such that $h\left(\mathbf{e}_{i}\right)=\mathbf{e}_{\sigma(i)}$ or $h\left(\mathbf{e}_{i}\right)=\overline{\mathbf{e}_{\sigma(i)}}$.

We want to show that the mapping $\sigma$ is injective. Suppose, on the contrary, that $\sigma(i)=\sigma(j)=s$ for some $i \neq j$. We first consider the case that $A$ is a field of characteristic $p>2$.

If $h\left(\mathbf{e}_{i}\right)=h\left(\mathbf{e}_{j}\right)=\mathbf{e}_{s}$, then $\mathbf{c}_{i}+\mathbf{d}=\mathbf{c}_{j}+\mathbf{d}=\mathbf{e}_{s}$ and so $\mathbf{c}_{i}=\mathbf{c}_{j}=\mathbf{e}_{s}-\mathbf{d}$. Then

$$
\begin{aligned}
h\left(\mathbf{e}_{i}+\mathbf{e}_{j}\right) & =\mathbf{c}_{i}+\mathbf{c}_{j}+\mathbf{d}=\mathbf{e}_{s}-\mathbf{d}+\mathbf{e}_{s}-\mathbf{d}+\mathbf{d} \\
& =\mathbf{e}_{s}+\mathbf{e}_{s}-\mathbf{d} \in\{0,1\}^{m} .
\end{aligned}
$$

But this implies that $\mathbf{d}=\mathbf{e}_{s}$ and so $w(\mathbf{d})=1$, a contradiction.

If $h\left(\mathbf{e}_{i}\right)=h\left(\mathbf{e}_{j}\right)=\overline{\mathbf{e}_{s}}$, we deduce as above that $h\left(\mathbf{e}_{i}+\mathbf{e}_{j}\right)=\overline{\mathbf{e}_{s}}+\overline{\mathbf{e}_{s}}-\mathbf{d} \in\{0,1\}^{m}$, which implies $\mathbf{d}=\overline{\mathbf{e}_{s}}$ and so $w(\mathbf{d})=m-1$, a contradiction.

If $h\left(\mathbf{e}_{i}\right)=\mathbf{e}_{s}$ and $h\left(\mathbf{e}_{j}\right)=\overline{\mathbf{e}_{s}}$, then $\mathbf{c}_{i}=\mathbf{e}_{s}-\mathbf{d}$ and $\mathbf{c}_{j}=\overline{\mathbf{e}_{s}}-\mathbf{d}$. Let $t$ be distinct from both $i$ and $j$, and assume that $h\left(\mathbf{e}_{t}\right)=\mathbf{e}_{\sigma(t)}$; then $\mathbf{c}_{t}=\mathbf{e}_{\sigma(t)}-\mathbf{d}$. Then

$$
\begin{aligned}
h\left(\mathbf{e}_{i}+\mathbf{e}_{j}+\mathbf{e}_{t}\right) & =\mathbf{e}_{s}-\mathbf{d}+\overline{\mathbf{e}_{s}}-\mathbf{d}+\mathbf{e}_{\sigma(t)}-\mathbf{d}+\mathbf{d} \\
& =\mathbf{1}+\mathbf{e}_{\sigma(t)}-(1+1) \mathbf{d} \in\{0,1\}^{m} .
\end{aligned}
$$


This implies that $\mathbf{d}=\mathbf{e}_{\sigma(t)}$ and so $w(\mathbf{d})=1$, a contradiction. Similarly, if $h(\mathbf{t})=$ $\overline{\mathbf{e}_{\sigma(t)}}$, then $h\left(\mathbf{e}_{i}+\mathbf{e}_{j}+\mathbf{e}_{l}\right)=\mathbf{1}+\overline{\mathbf{e}_{\sigma(t)}}-(1+1) \mathbf{d} \in\{0,1\}^{m}$, which implies that $\mathbf{d}=\overline{\mathbf{e}_{\sigma(t)}}$, a contradiction.

Consider then the case that $A$ has characterictic $p=2$. Let $t$ be distinct from both $i$ and $j$, and assume that $h\left(\mathbf{e}_{i}\right)=h\left(\mathbf{e}_{j}\right)=\mathbf{e}_{s}, h(\mathbf{t})=\mathbf{e}_{\sigma(t)}$. Then

$$
h\left(\mathbf{e}_{i}+\mathbf{e}_{j}+\mathbf{e}_{t}\right)=\mathbf{e}_{s}-\mathbf{d}+\mathbf{e}_{s}-\mathbf{d}+\mathbf{e}_{\sigma(t)}-\mathbf{d}+\mathbf{d}=\mathbf{e}_{\sigma(t)},
$$

which is a contradiction. Assume then that $h\left(\mathbf{e}_{i}\right)=\mathbf{e}_{s}, h\left(\mathbf{e}_{j}\right)=\overline{\mathbf{e}_{s}}, h(\mathbf{t})=\mathbf{e}_{\sigma(t)}$. Then

$$
h\left(\mathbf{e}_{i}+\mathbf{e}_{j}+\mathbf{e}_{t}\right)=\mathbf{e}_{s}-\mathbf{d}+\overline{\mathbf{e}_{s}}-\mathbf{d}+\mathbf{e}_{\sigma(t)}-\mathbf{d}+\mathbf{d}=\mathbf{1}+\mathbf{e}_{\sigma(t)}=\overline{\mathbf{e}_{\sigma(t)}},
$$

again a contradiction. The other cases where $\mathbf{e}_{i}, \mathbf{e}_{j}, \mathbf{e}_{t}$ are mapped to the various unit vectors and complements of unit vectors lead similarly to a contradiction.

We conclude that the mapping $\sigma$ is injective. Thus, if $m<n$, there is no such mapping and therefore $\alpha_{n} \npreceq_{L} \alpha_{m}$. We now assume that $n<m$ and $n$ and $m$ are congruent to 2 modulo $p$.

Let $S_{1}=\left\{i \in\{2, \ldots, n\}: h\left(\mathbf{e}_{i}\right)=\mathbf{e}_{\sigma(i)}\right\}$ and $S_{2}=\left\{i \in\{2, \ldots, n\}: h\left(\mathbf{e}_{i}\right)=\right.$ $\left.\overline{\mathbf{e}_{\sigma(i)}}\right\}$. Define the $m$-vector $\mathbf{v}=\left(v_{1}, \ldots, v_{m}\right)=h\left(\overline{\mathbf{e}_{1}}\right)$. Taking into account that $\mathbf{d} \in\{0,1\}^{m}$ and that the characteristic $p$ of $A$ divides $n-2$, we have that

$$
\begin{aligned}
h\left(\overline{\mathbf{e}_{1}}\right)=\mathbf{d}+\sum_{i=2}^{n} \mathbf{c}_{i} & =\mathbf{d}+\sum_{i \in S_{1}}\left(\mathbf{e}_{\sigma(i)}-\mathbf{d}\right)+\sum_{i \in S_{2}}\left(\overline{\mathbf{e}_{\sigma(i)}}-\mathbf{d}\right) \\
& =\sum_{i \in S_{1}} \mathbf{e}_{\sigma(i)}+\sum_{i \in S_{2}} \overline{\overline{\mathbf{e}}_{\sigma(i)}} .
\end{aligned}
$$

If both $S_{1}$ and $S_{2}$ are nonempty, let $r \in S_{1}, s \in S_{2}$ and $t \notin \operatorname{Im} \sigma$. Then $v_{\sigma(r)}$, $v_{\sigma(s)}$ and $v_{t}$ are distinct elements of $A$, and so $\mathbf{v} \notin\{0,1\}^{m}$. If either $S_{1}$ or $S_{2}$ is empty, then $\mathbf{v} \in\{a, b\}^{m}$ for some $a, b \in A$, but $w(\mathbf{v}) \notin\{1, m-1\}$. We have reached a contradiction, because $\mathbf{v}$ should be a unit vector or the complement of a unit vector. We conclude that $\alpha_{m} \|_{L} \alpha_{n}$.

Theorem 5.10. There is an infinite antichain of $L$-incomparable functions.

\section{The ClONES OF MONOTONE FUnCTIONS}

6.1. Order-preserving maps between posets. Let $(P, \leq),(Q, \leq)$ be posets. A mapping $f: P \rightarrow Q$ is order-preserving (or monotone) if $a \leq b$ in $P$ implies $f(a) \leq f(b)$ in $Q$. A mapping $f$ is order-reflecting, if $f(a) \leq f(b)$ in $Q$ implies $a \leq b$ in $P$. An order-embedding is a map that is both order-preserving and orderreflecting. Posets $P$ and $Q$ are order-equivalent, if there are order-preserving maps $f: P \rightarrow Q$ and $g: Q \rightarrow P$. An order-isomorphism is an order-preserving bijection whose inverse is also order-preserving; or, equivalently, a surjective orderembedding. Posets $P$ and $Q$ are isomorphic, denoted $P \cong Q$, if there is an orderisomorphism of $P$ to $Q$. Isomorphic posets are order-equivalent by definition. The composition of order-preserving maps is order-preserving.

A subposet $Q$ of a poset $P$ is a retract of $P(P$ retracts to $Q)$ if there is an order-preserving map $g: P \rightarrow Q$ whose restriction to $Q$ equals the identity map on $Q$. We also say that a poset $Q$ is a retract of a poset $P$ if there are order-preserving maps $f: Q \rightarrow P$ and $g: P \rightarrow Q$ such that $g \circ f$ is the identity map on $Q$. In either case $g$ is called a retraction map; in the latter case $f$ is called a coretraction map. One can regard the former definition as a special case of the latter: the inclusion 
map $Q \rightarrow P$ can be chosen as the coretraction. A retract $Q$ of $P$ is proper if it is not isomorphic to $P$. The composition of retractions is again a retraction.

A family $\left(P_{i}: i \in I\right)$ of posets is a representation of a poset $P$ if each $P_{i}$ is a retract of $P$ and $P$ itself is a retract of the direct product $\prod_{i \in I} P_{i}$. It is known (see $[2,14])$ that every complete lattice $L$ with at least two elements has a representation $\left(P_{x}: x \in L\right)$, where each $P_{x} \cong \mathbf{2}$. It is also well-known that for every poset $P$ that is not an antichain, $P^{n}$ retracts to $\mathbf{2}^{n}$. Thus, for any finite lattice $L$ and any poset $P$ that is not an antichain, there exists an integer $n$ such that $L$ is a retract of $P^{n}$.

6.2. Coloured posets. An $S$-coloured poset, or an $S$-poset, is an object $((P, \leq), f)$, where $(P, \leq)$ is a partially ordered set (the underlying poset) and $f: P \rightarrow S$ is a colouring function. If $(Q, \leq)$ is a subposet of $(P, \leq)$, then $\left((Q, \leq),\left.f\right|_{Q}\right)$ is an $S$-coloured poset, and it is called an $S$-coloured subposet, or an $S$-subposet, of $((P, \leq), f)$. An $S$-coloured poset whose underlying poset is a lattice is called an $S$-coloured lattice or an $S$-lattice. The set of all $S$-coloured posets is denoted by $\mathcal{P}_{S}$. We usually deal with colouring functions with codomain $S=\{0,1, \ldots, k-1\}=\underline{k}$, and we call a $\underline{k}$-coloured poset (lattice) a $k$-poset ( $k$-lattice).

Let $((P, \leq), f)$ and $((Q, \leq), g)$ be $S$-posets. A mapping $h: P \rightarrow Q$ is colourpreserving, if $f=g \circ h$. A homomorphism of $P$ to $Q$ is a map $h: P \rightarrow Q$ that is both order-preserving and colour-preserving. We define a preorder $\leq$ on the set $\mathcal{P}_{S}$ of all $S$-coloured posets as follows: $P \leq Q$ if and only if there exists a homomorphism of $P$ to $Q$. The induced equivalence relation on $\mathcal{P}_{S}$ is denoted by $\equiv$. Information on $k$-posets and their homomorphicity order may be found in the works by Kosub [8], Kosub and Wagner [9], and Selivanov [17].

A bijective homomorphism is called an isomorphism. Coloured posets $P$ and $Q$ are isomorphic, denoted $P \cong Q$, if there exists an isomorphism between $P$ and $Q$.

A coloured subposet $Q$ of a coloured poset $P$ is a retract of $P$, denoted $Q \triangleleft$ $P$, if there is a retraction map $h: P \rightarrow Q$ between the underlying posets that is also colour-preserving. We also say that a coloured poset $Q$ is a retract of a coloured poset $P$, also denoted $Q \triangleleft P$, if there are colour-preserving retraction and coretraction maps $h: P \rightarrow Q$ and $k: Q \rightarrow P$. If $Q \triangleleft P$, then $P \equiv Q$ by definition.

If $h$ is a retraction map from a poset $P$ to a poset $Q$ and $g$ is a colouring function of $Q$, then $g \circ h$ is a colouring function of $P$ and the coloured poset $(P, g \circ h)$ retracts to $(Q, g)$.

For $n, m \in \mathbb{N}$, denote by $\mathcal{L}(m, n)$ the 3 -lattice depicted in Figure 1 . Kosub and Wagner [9] pointed out that for any $m, n \in \mathbb{N}, \mathcal{L}(m+1, n)<\mathcal{L}(m, n)$, and for $m \neq n, \mathcal{L}(m, m) \| \mathcal{L}(n, n)$. Thus, there are both an infinite descending chain and an infinite antichain of 3-lattices.

6.3. Monotone subfunctions. Assume that the base set $A$ is equipped with a partial order $\leq$. A function $f: A^{n} \rightarrow A$ is monotone with respect to $\leq$, if $f(\mathbf{x}) \leq$ $f(\mathbf{y})$ whenever $\mathbf{x} \leq \mathbf{y}$. The class $M_{\leq}$of monotone functions with respect to $\leq$is a clone on $A$. Furthermore, if $(A, \leq)$ is bounded, then $M_{\leq}$is a maximal clone by Rosenberg's classification [15].

We associate with any $n$-ary function $f$ on $A$ the $A$-coloured poset $P(f, \leq)=$ $\left((A, \leq)^{n}, f\right)=\left(\left(A^{n}, \leq^{\prime}\right), f\right)$, with the component-wise order $\leq^{\prime}$.

Proposition 6.1. $f \preceq_{M_{\leq}} g$ if and only if $P(f, \leq) \leq P(g, \leq)$. 


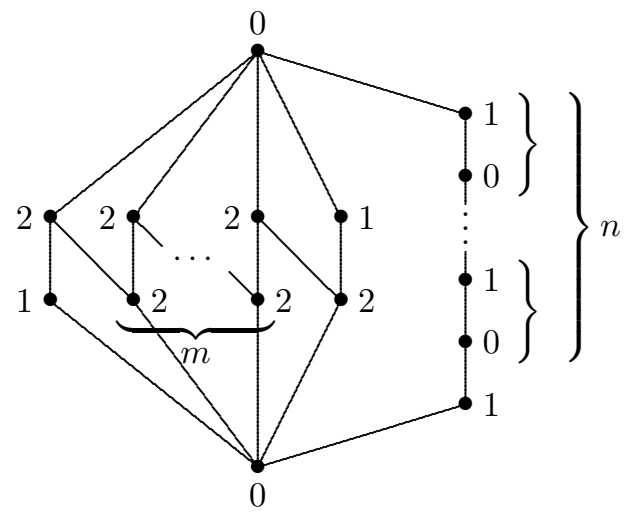

Figure 1. The 3-lattice $\mathcal{L}(m, n)$.

Proof. If $f=g\left(h_{1}, \ldots, h_{m}\right)$, where $h_{1}, \ldots, h_{m} \in M_{\leq}$, then $h=\left(h_{1}, \ldots, h_{m}\right)$ is a homomorphism of $P(f, \leq)$ to $P(g, \leq)$. Conversely, the components of any homomorphism $h: P(f, \leq) \rightarrow P(g, \leq)$ are functions in $M_{\leq}$and $f=g \circ h$.

Theorem 6.2. Assume that $|A| \geq 3$ and the poset $(A, \leq)$ is not an antichain. Then there is an infinite descending chain of $M_{\leq}$-subfunctions and an infinite antichain of $M_{\leq}$-incomparable functions.

Proof. By the previous observations, for every $A$-coloured lattice $L$, there exists an integer $n$ and a function $f: A^{n} \rightarrow A$ such that $L \triangleleft P(f, \leq)$. There exists an infinite descending chain and an infinite antichain of 3 -lattices. Since a coloured poset is homomorphically equivalent to all its retracts, we deduce that there exists an infinite descending chain of $M_{\leq}$-subfunctions and an infinite antichain of $M_{\leq-}$ incomparable functions, provided that $|A| \geq 3$.

Note that if $(A, \leq)$ is an antichain, then $M_{\leq}=\mathcal{O}_{A}$, because monotonicity with respect to an antichain imposes no restrictions whatsoever on functions. $\mathcal{O}_{A}$-subfunctions are examined in Section 3.

\section{Conclusions}

We have analyzed the $\mathcal{C}$-subfunction relations defined by certain clones on a finite set $A$ with $|A|=k \geq 3$, namely by the clone $\mathcal{O}_{A}$ of all functions on $A$; by the clones $\langle M\rangle$ generated by transformation monoids $M$ on $A$; by the clones $L$ (and $L_{0}$ ) of linear functions (with constant term $\widehat{0}$ ) on a finite field; and by the clones $M_{\leq}$of monotone functions with respect to a partial order $\leq$ on $A$.

The poset $\left(\mathcal{O}_{A} / \equiv_{\mathcal{O}_{A}}, \leq_{\mathcal{O}_{A}}\right)$ is isomorphic to $(\mathcal{P}(\bar{A}) \backslash\{\emptyset\}, \subseteq)$. The largest chain of this poset has $k$ elements, and the largest antichain has $\left(\begin{array}{c}k \\ \lfloor k / 2\rfloor\end{array}\right)$ elements. The $\langle M\rangle$-, $L$-, and $L_{0}$-subfunction relations do not have infinite descending chains but they do have infinite antichains. These observations conform with the results we obtained for the corresponding Boolean subfunctions in [10].

The $M_{\leq}$-subfunction relation has both infinite descending chains and infinite antichains. This is in sharp contrast with the case where $|A|=2$. Namely, we have established in [10] that the subfunction relation defined by the clone of monotone 
Boolean functions has no infinite descending chains and the largest antichain has only two elements.

\section{REFERENCES}

[1] Davey, B. A. and Priestley, H. A.: Introduction to Lattices and Order, Second edition, Cambridge University Press, Cambridge, 2002.

[2] Duffus, D. and Rival, I.: A structure theory for ordered sets, Discrete Math. 35 (1981), 53-118.

[3] Grätzer, G.: General Lattice Theory, second edition, Birkhäuser, Basel, 2003.

[4] Green, J. A.: On the structure of semigroups, Ann. of Math. (2) 54 (1951), 163-172.

[5] Henno, J.: Green relations in Menger systems, Tartu Riikl. Ül. Toimetised 277 (1971), 37-46 (in Russian, with Estonian and English summaries).

[6] Henno, J.: On idempotents and Green relations in the algebras of many-placed functions, Ann. Acad. Sci. Fenn. Ser. A I Math. 3 (1977), 169-178.

[7] Howie, J. M.: Fundamentals of Semigroup Theory, London Mathematical Society Monographs, New Series 12, Oxford University Press, 1995.

[8] Kosub, S.: NP-partitions over posets with an application to reducing the set of solutions of NP problems, Theory Comput. Systems 38 (2005), 83-113.

[9] Kosub, S. and Wagner, K. W.: The Boolean hierarchy of NP-partitions, In: H. Reichel and S. Tison (eds.), STACS 2000, 17th Annual Symposium on Theoretical Aspects of Computer Science, Lecture Notes in Comput. Sci. 1770, Springer-Verlag, Berlin, 2000, pp. 157-168. An expanded version is available as Technical Report TUM-I0209, Institut für Informatik, Technische Universität München, München, 2002.

[10] Lehtonen, E.: Order-theoretical analysis of subfunction relations between Boolean functions, manuscript, 2005, http://math.tut.fi/algebra/.

[11] Menger, K.: The algebra of functions: past, present, future, Rend. Mat. Appl. 20 (1961), 409-430.

[12] Pippenger, N.: Galois theory for minors of finite functions, Discrete Math. 254 (2002), 405419.

[13] Post, E. L.: The Two-Valued Iterative Systems of Mathematical Logic, Annals of Mathematical Studies 5, Princeton University Press, Princeton, 1941.

[14] Pouzet, M. and Rival, I.: Every countable lattice is a retract of a direct product of chains, Algebra Universalis 18 (1984), 295-307.

[15] Rosenberg, I.: Über die funktionale Vollständigkeit in den mehrwertigen Logiken, Rozpravy Československé Akad. Věd, Ǩada Mat. Přŕrod. Věd 80 (1970), 3-93.

[16] Schein, B. M. and Trohimenko, V. S.: Algebras of multiplace functions, Semigroup Forum 17 (1979), 1-64.

[17] Selivanov, V. L.: Boolean hierarchies of partitions over a reducible base, Algebra Logic 43 (2004), 44-61. Translated from Algebra Logika 43 (2004), 77-109.

[18] Sperner, E.: Ein Satz über Untermengen einer endlichen Menge, Math. Z. 27 (1928), 544-548.

[19] Szendrei, Á.: Clones in Universal Algebra, Séminaire de mathématiques supérieures 99, Les Presses de l'Université de Montréal, Montréal, 1986.

[20] Wang, C.: Boolean minors, Discrete Math. 141 (1991), 237-258.

[21] Zverovich, I. E.: Characterizations of closed classes of Boolean functions in terms of forbidden subfunctions and Post classes, Discrete Appl. Math. 149 (2005), 200-218.

Institute of Mathematics, Tampere University of Technology, P.O. Box 553, FI-33101

TAMPERE, FinLAND

E-mail address: erkko.lehtonen@tut.fi 